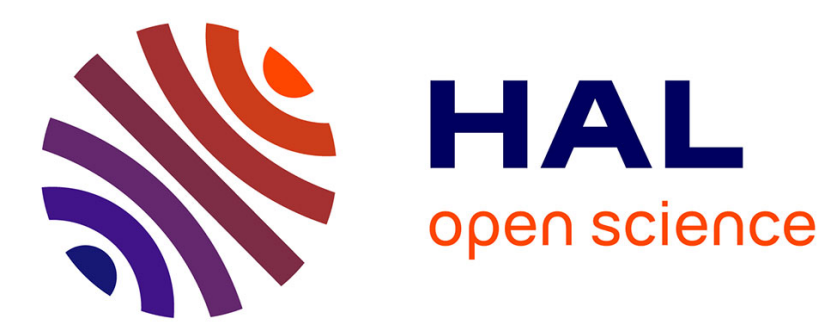

\title{
How to render Neural Fields more realistic
}

Axel Hutt, Meysam Hashemi, Peter Beim Graben

\section{To cite this version:}

Axel Hutt, Meysam Hashemi, Peter Beim Graben. How to render Neural Fields more realistic. 2014. hal-01007681

\section{HAL Id: hal-01007681 \\ https://inria.hal.science/hal-01007681}

Preprint submitted on 17 Jun 2014

HAL is a multi-disciplinary open access archive for the deposit and dissemination of scientific research documents, whether they are published or not. The documents may come from teaching and research institutions in France or abroad, or from public or private research centers.
L'archive ouverte pluridisciplinaire HAL, est destinée au dépôt et à la diffusion de documents scientifiques de niveau recherche, publiés ou non, émanant des établissements d'enseignement et de recherche français ou étrangers, des laboratoires publics ou privés. 


\section{How to render Neural Fields more realistic}

Axel Hutt and Meysam Hashemi and Peter beim Graben

\section{Introduction}

Complex systems are omnipresent in nature. They exhibit multiple spatial and temporal scales whose understanding and control is one of the most challenging problems of research for the last centuries. The notion of complexity is not welldefined [1], but typically a system is said to be complex if, in addition to the multiple scales, there exist self-organised sub-units in the system which are generated by smaller units. In many biological systems, climbing this hierarchy from smaller units to larger units implies increasing the spatial and temporal scale.

Before discussing neural systems, we would like to clarify a major scientific problem in this context: the choice of the description level. Let us assume the task to describe a water wave mathematically, i.e., derive a mathematical model whose solution describes the spatiotemporal phenomenon of a wave. Some researchers may ask: we want to understand how water works, and especially, how a wave is generated. Hence, a straightforward simple and fundamental approach is to take a closer look at the building blocks of water (remark : this is very similar to the way how biologists work in neuroscience today). The researchers find $\mathrm{H}_{2} \mathrm{O}$-molecules, study the properties of the atoms and the inter-molecule interactions like hydrogen bridges and van-der-Waals bounds. Now, to describe a water wave, one has to study millions and millions of these molecules and their interactions. To this end, the researchers

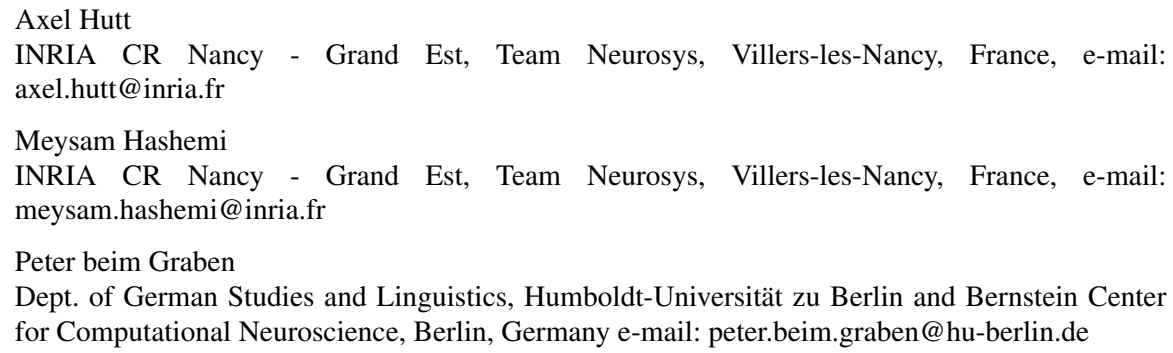


take the reasonable approach to start simulating two, three, then and even hundreds of these molecules. The numerical analysis is demanding, but still there is no description of the water waves, since the system appears to be so complex taking into account all the different interactions of molecules and the research in this hard task got stuck in some way.

Of course it is well-known that it is not necessary to study single molecules to describe water waves, today we take the famous Navier-Stokes equation (NSE) which includes the solution for this phenomenon. This equation involves mean fluid properties, e.g., inner friction and viscosity [2]. Hence the NSE considers average properties of interacting single molecules. In other words, it does not know single molecules, but provides a powerful description of a large ensemble or mass of molecules. It allows to describe several different rather complex fluid phenomena, but of course no phenomena related to single molecules. Hence the NSE equation provides a very good mathematical description of the system at a macroscopic description level only. Going back to the intended description of water waves by a single molecule study, this approach is not reasonable, probably it will not lead to a good description level of macroscopic phenomena and hence it is not constructive.

In todays' neuroscience, the approach of linking single neuron activity to macroscopic phenomena is attractive, e.g. in the context of cognition [3-5], sleep [6] or anaesthesia $[7,8]$. These studies state a relationship between the single neuron activity (microscopic scale) and behavioral phenomenon (macroscopic scale). However, to our best knowledge it is not understood how the different experimental findings on different scales are linked to each other, i.e., the link between the two scales is not understood and no model linking the scales has been developed yet. This situation resembles closely the water wave task described above: it is clear that there is a relation between small sub-units (molecules or neurons) to large complex systems units (water wave or cognition), since the dynamics of the large units is generated by the sub-units, but a link appears to be too complex. Consequently, learning from physics and the NSE, it is necessary to consider more abstract, intermediate models whose elements are based on small sub-units properties but which allow to model large unit phenomena. In other words, it is much more effective to consider mesoscopic population models involving average properties of interacting neurons and which allow to describe macroscopic experimental phenomena, such as Local Field Potentials (LFP), encephalographic activity (EEG/MEG) or even behaviour. Promising candidates for such models are neural mass or neural field models. The present book chapter discusses recent advances in these models rendering the standard neural population models more realistic.

The subsequent sections do not give a complete overview over the recent advances in the field, a recent excellent review article already provides this information [9]. The present chapter first introduces briefly to two types of neural field models. Then it gives some details of few selected extensions, always introducing the neuroscientific problem by experimental data before presenting a mathematical description of the phenomena described. 


\section{Two classes of neural field models}

\subsection{Amari model}

The first neural field models developed by Wilson and Cowan [10] and Amari [11] are continuum limits of large-scale neural networks. Typically, their dynamic variables describe either mean voltage [11] or mean firing rate $[10,12]$ of a population element of neural tissue, see also the excellent review article of Bressloff [9]. In some subsequent sections we consider the paradigmatic Amari equation [11] describing the spatiotemporal dynamics of mean potential $V(x, t)$ over a cortical $d$ dimensional manifold $\Omega \subset \mathbf{R}^{d}$ :

$$
\tau \frac{\partial V(x, t)}{\partial t}=-V(x, t)+\int_{\Omega} K(x, y) S[V(y, t)] \mathrm{d} y+I(x, t) .
$$

with the spatial synaptic kernel $K(x, y)$ which defines the connectivity between site $y \in \Omega$ and site $x \in \Omega$. The transfer function $S$ is nonlinear and typically of sigmoidal shape. This model considers external inputs $I(x, t)$, e.g., originating from other extracortical populations and from external stimulation. The model (1) takes into account a single synaptic time scale $\tau$ assuming an exponential synaptic response function. However we point out that re-scaling of time allows to set $\tau=1$.

In general, the connectivity kernel $K(x, y)$ fully depends on both sites $x$ and $y$ reflecting spatial heterogeneity. If the connectivity solely depends on the difference between $x$ and $y$, i.e. $K(x, y)=K(x-y)$, then the neural field activity does not depend on specific spatial locations and hence is translational invariant. This case is called spatially homogeneity [11]. If the connectivity even depends on the distance between $x$ and $y$ only, i.e. $K(x, y)=K(\|x-y\|)$, with $\|x\|$ as some norm in $\Omega$, then the neural field is spatially homogeneous and isotropic [13].

Several extensions of the Amari model (1) are possible, such as the consideration of finite axonal transmission speeds $[14,15]$, constant feedback delays $[16,17]$ (see also section 3 ), heterogeneity [18, 19] (see also section 4), spike-frequency adaption [20], statistical properties of single neurons [21], the combination of several brain areas [22], electromagnetic fields [23, 24] and many more [9].

Mathematically, Eq. (1) is an integro-differential equation. Spatially homogeneous (respectively isotropic) neural fields have been intensively studied in the literature due to their nice analytical properties $[14,15,25,26]$. Moreover, these models may be transformed to derive partial differential wave equations $[25,27,28]$ for certain classes of synaptic kernels.

\subsection{Robinson model}

As mentioned at the end of the previous paragraph, under certain conditions integrodifferential equations may be transformed to partial differential equations. In the 
1990's James Wright and David Liley started developing partial differential equation models for neural population activity [29]. Their work has inspired other teams, e.g., Peter Robinson and colleagues, who developed a similar neural model which has been proven to be very successful. This type of neural field model [30-32] is based on a population-level model of a single thalamo-cortical module consisting of excitatory (E) and inhibitory (I) cortical population, thalamic relay neurons (S), and thalamic reticular neurons (R). The average soma membrane potential is modeled by

$$
V_{a}(t)=\sum_{b=E, I, R, S} \bar{h}(t) \otimes K_{a, b} \phi_{b}\left(t-\tau_{a, b}\right), a=E, I, S, R
$$

where $\otimes$ denotes temporal convolution and $\phi_{b}$ is the pulse firing rate of the population $b$. The constants $K_{a, b}$ are the strengths of the connections from population of type $b$ to population of type $a$. The delay term, $\tau_{a, b}$ is zero for intra-cortical and intra-thalamic connections and non-zero for thalamocortical or corticothalamic connections [31].

The model assumes that only axons of excitatory cortical neurons are long enough to emit axonal propagating pulses. Moreover, $\phi_{E}$ obeys the damped oscillator equation

$$
D \phi_{E}=S\left(V_{a}\right)
$$

with the operator $D$

$$
D=\left(\frac{1}{\gamma} \frac{\partial}{\partial t}+1\right)^{2}
$$

In Eq. (2) $\bar{h}(t)$ denotes the mean synaptic response function

$$
\bar{h}(t)=\frac{\alpha \beta}{\beta-\alpha}\left(e^{-\alpha t}-e^{-\beta t}\right),
$$

where $\alpha$ and $\beta$ are the synaptic decay and rise rate of synaptic response function, respectively.

\section{Delayed nonlocal feedback between populations}

In neural fields, one might include delays in several ways. The finite axonal transmission delay is proportional to the fraction of distance between two spatial locations and transmission speed and takes into account the finite propagation speed of action potentials along axonal branches [33], or in more general terms, it considers the finite-time interaction between two elements in a spatially extended system [28]. In addition, one could argue that delayed interactions happen on a single-neuron scale between neurons and it is more reasonable to treat these inter-neuron delays as a kind of effective delay [17,34]. This latter type of delay is constant. In addition to these two delay types, the nonlocal feedback delay takes into account the finite axonal transmission speed along axonal pathways between two brain areas. Since 
this axonal pathway has a finite defined length, the transmission delay is fixed and hence also constant [16]. Variations of all these delay types may be considered by distributed transmission speeds and/or distributed delays [15,35]. The subsequent paragraphs consider constant delays reflecting the finite transmission speed along axonal branches between brain areas.

\subsection{The primary sensory area in weakly-electric fish}

To examine the neural decoding in weakly electric fish, Doiron et al. [36] had performed an experimental in vivo stimulation study. A dipole was placed near the skin of a fish to stimulate only a part of the receptive field. Figure 1 sketches the experimental setup, the spike autocorrelation $A(t)$ and the interspike interval (ISI) histograms of a typical pyramidal cell response to a local and global stimulus. The stimulus was temporal noise evoking spatially weakly correlated sensory receptor activity for local stimulation and strong spatial correlations in the receptor dynamics in case of the experimentally global stimulation. The global random stimulus evokes bursting in the neuron activity whereas the local stimulus evokes a single principal firing mode. This experiment raises the question how the spatial correlations in the input stimulus interact with those imposed by the physiological system.
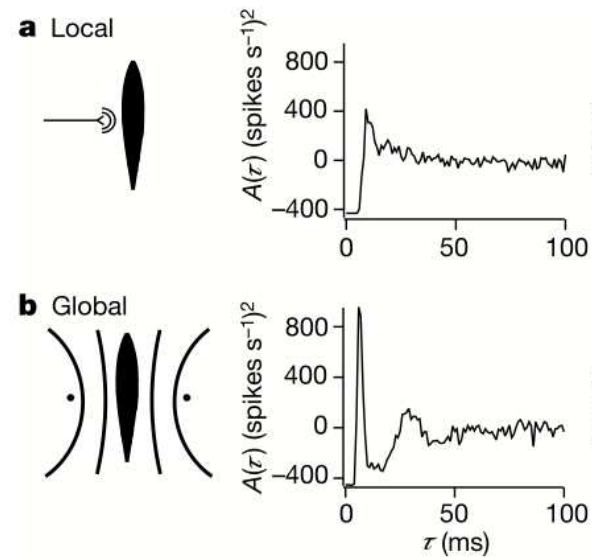
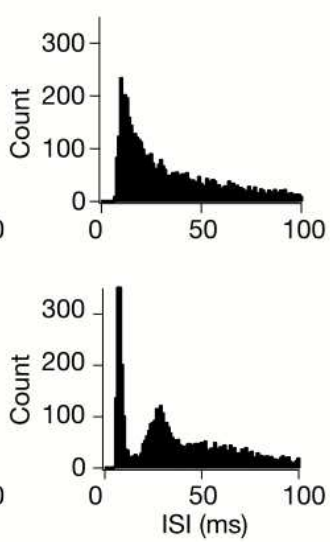

Fig. 1 Experimental setup and firing statistics of stimulation experiment [36]. In (a), the electric skin stimulation is local (left panel) and induces a single main oscillation as seen in the spike autocorrelation function $A(\tau)$ (center panel) and the corresponding histogram (right panel). In (b) the stimulus was global inducing an additional oscillation mode. Taken from [36] by permission.

A rather simple population model considers the primary sensory areas in the electro-sensory system of weakly electric fish [37], but similar configurations can also be found in parts of the vertebrate brain [38]. The model sketched in Fig. 2 [39] 
is made up of the ELL, a layer of pyramidal cells driven by the primary receptors that receive an external stimulus, and the higher area $N p$. These areas are spatially coupled via a delayed topographic feedback with connectivity kernels $K_{e n}(x)$ and $K_{n e}(x)$ which reflect connections from the $N p(n)$ to the $E L L(e)$ and vice versa, respectively, see Fig. 2 . The neurons in both populations have insignificant direct couplings and the coupling from the ELL to $N p$ is excitatory and delayed in time by $\tau_{1}$, the coupling back to the ELL is inhibitory with delay $\tau_{2}$. Moreover, according to the experimental setup in [36], the model considers excitatory spatiotemporal stimuli $I(x, t)$ to the ELL.

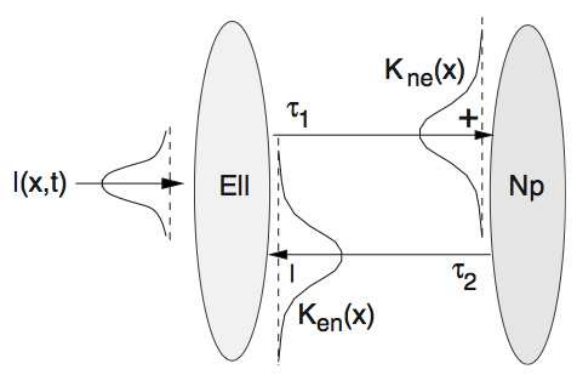

Fig. 2 Topography of the delayed feedback model. The plus and minus signs indicate the excitatory and inhibitory connections, respectively.

The aim of the study is to learn more about the mechanism how to change the principal oscillation frequency by the properties of an external stimulus. The model considers spatiotemporal noise with a well-defined and adjustable spatial correlation length. The population model [39] describes two coupled neural fields whose activities are strongly related to experimentally observable local field potentials [40].

Then the theoretical power spectrum in the ELL reads

$$
P(v)=\int_{-\infty}^{\infty} R(v, l) \tilde{C}(l) d l
$$

where $R(v, l)$ is the spectral response function and $\tilde{C}(l)$ is the scaled Fourier transform of the input correlation function. It turns out, that the power spectrum (6) does not depend on the spatial scale of the feedback loop $\sigma_{f}$ and the input correlation scale $\sigma_{i}$ independently, but just depends on their ratio, called $\eta$. This finding reflects the coupling of the spatial scale of the external input to the intrinsic spatial scale of the system.

Figure 3 shows the resulting power spectra for two values of the spatial scale ratio $\eta$. We observe that a small ratio $\eta=\sigma_{f} / \sigma_{i} \ll 1$ generates a spectral peak at about $20 \mathrm{~Hz}$, whereas the large ratio $\eta \gg 1$ generates a power peak at about $0 \mathrm{~Hz}$. Hence retaining the topographic feedback but decreasing the input correlation function from large values of $\sigma_{i}=\sigma_{f} / \eta$ (global noise) to small values of $\sigma_{i}$ (local noise) switches the spectral peak, similar as observed experimentally by Doiron et al. [36]. 


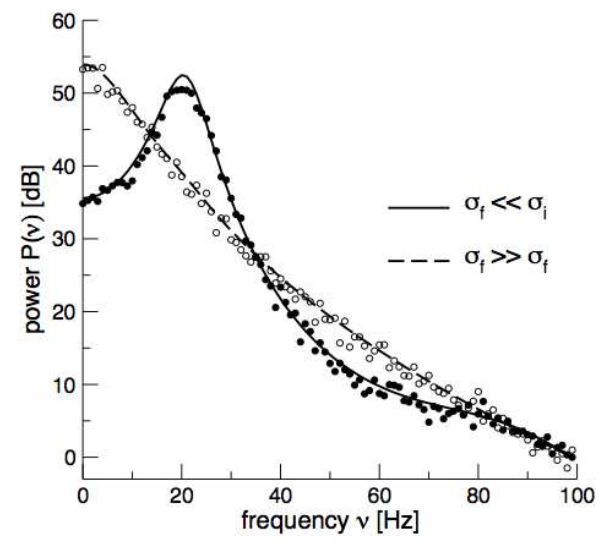

Fig. 3 Theoretical power spectrum computed for $\eta=40$ (solid line) and $\eta=1 / 40$ (dashed line).

(a)

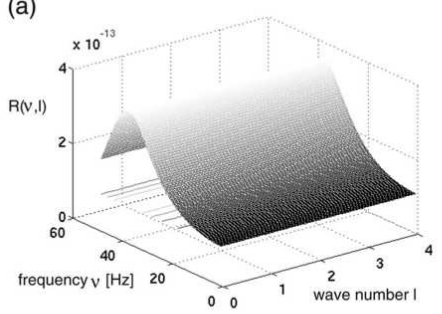

(c)

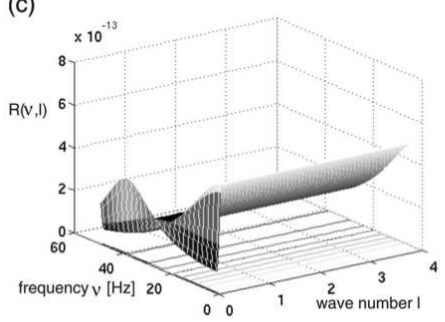

(b)

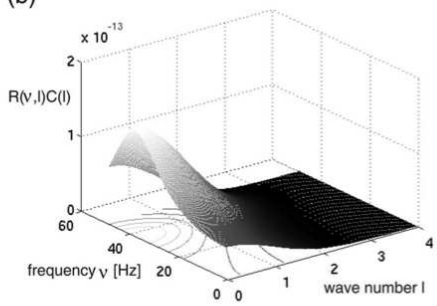

(d)

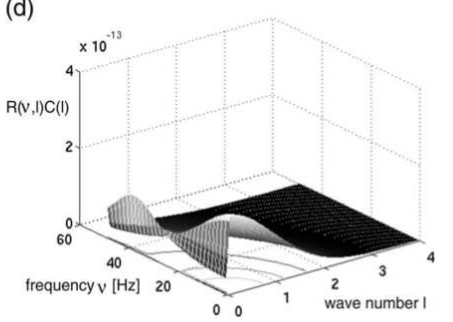

Fig. 4 The response function $R(v, l)$ and the integrand of the power spectrum integral $R(v, l) \tilde{C}(l)$ for (a,b) $\sigma_{i}=40 \sigma_{f}$ (global noise), (c,d) $\sigma_{i}=\sigma_{f} / 40$ (local noise). Taken from [39] by permission.

To understand this, Figure 4 shows the response function $R(v, l)$ and the integrand $R\left(v_{l}\right) \tilde{C}(l)$ in the definition of the power spectrum (6). The response function $R$ and $R \tilde{C}$ have a single maximum at about $20 \mathrm{~Hz}$ for global noise (Fig. 4(a,b)), whereas $R$ and $R \tilde{C}$ have two local maxima at $20 \mathrm{~Hz}$ and $0 \mathrm{~Hz}$ (Fig. 4(c,d)). Since the peak of $R \tilde{C}$ at $0 \mathrm{~Hz}$ is broader than the peak about $20 \mathrm{~Hz}$ and the power spectrum is the integral over $R(\tilde{l})$, cf. Eq. (6), the contribution of $R \tilde{C}$ to the power at $0 \mathrm{~Hz}$ exceeds the contribution at $20 \mathrm{~Hz}$ yielding a strong peak at $0 \mathrm{~Hz}$. 
This result reveals that the spectral peak in Fig. 3 at $0 \mathrm{~Hz}$ results from the selection of one mode out of two possible modes at frequencies of $0 \mathrm{~Hz}$ and $20 \mathrm{~Hz}$, whereas the spectral peak at $20 \mathrm{~Hz}$ is the only oscillation mode present in the system. The switch between these two configurations depends on the spatial correlation of the stimulus noise. These modes reflect activity subnetworks from which only one is engaged.

\subsection{General anaesthesia}

General anaesthesia is an important medical application in today's hospital surgery, but its underlying neural interactions is still a mystery. In the last decades, general anaesthesia has attracted theoretical researchers [41-45]. Most theoretical studies aim to explain signal features of electroencephalographic data (EEG) observed during anaesthesia, such as the attenuation or enhancement of $\alpha$-activity accompanied by a subsequent enhancement of $\delta$-activity while increasing anaesthetic concentration [46, 47], cf. Fig. 5. The subsequent paragraphs show how neural field models
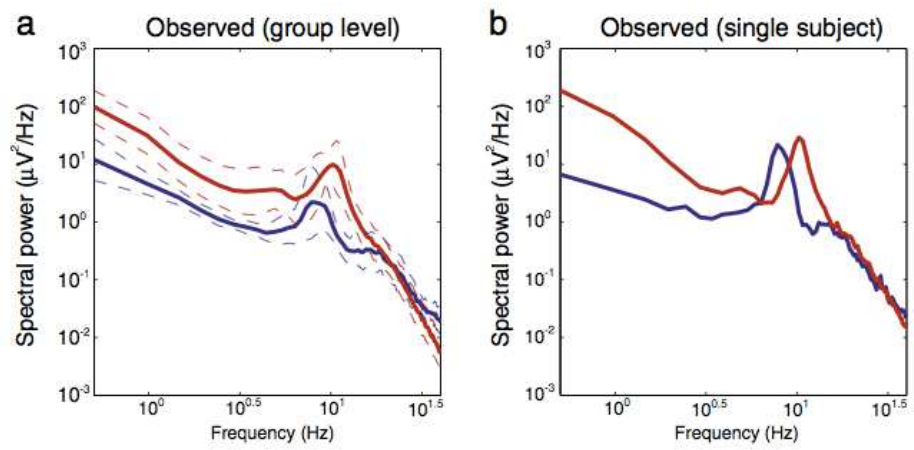

Fig. 5 The power spectra measured in frontal EEG electrodes in the absence of anaesthesia (blue line) and during propofol anaesthesia (red line) in a group of subjects (a) and for a single subject (b). Taken from [30] by permission.

may explain on the power enhancement and the frequency shift of maximum power while increasing the anaesthetic concentration.

To this end, we consider a derivative of the Robinson model introduced in section 2.2 and introduce a new sigmoid function derived from properties of type-I neurons [48]

$$
S\left(V_{a}\right)=F\left(V_{a}, 0\right)-F\left(V_{a}, \gamma\right),
$$

with

$$
F\left(V_{a}, \gamma\right)=\frac{S_{\max }}{2}\left(1+\operatorname{erf}\left(\frac{V_{a}-\theta-\gamma \sigma^{2}}{\sqrt{2} \sigma}\right)\right) e^{-\gamma\left(V_{a}-\theta\right)+\gamma^{2} \sigma^{2} / 2}
$$


in which the parameter $\gamma<\infty$ takes into account the properties of type I-neurons, $S_{\max }$ is the maximum population firing rate, $\theta$ is the mean firing threshold, and $\sigma$ is related to the standard deviation of firing thresholds in the populations.

External input to the system can be considered as a non-specific input to relay neurons as

$$
\phi_{N}=\left\langle\phi_{N}\right\rangle+\sqrt{2} \kappa \xi(t)
$$

where $\left\langle\phi_{N}\right\rangle$ is its mean value and $\xi(t)$ is Gaussian white noise of strength $\kappa$ with zero mean.

It is well-known that general anaesthetics (GA) bind directly to sensitive target receptors. For instance, a large number of studies link the effect of many GAs to altered function of the $\mathrm{GABA}_{\mathrm{A}}$ receptors. Recent clinical findings have revealed several sites of elicited anaesthetic action of the anaesthetic propofol in the human brain, e.g., propofol suppresses field potentials in the rat thalamus and cortex [49].

In detail, the anaesthetic propofol increases the decay time constant of synaptic $\mathrm{GABA}_{\mathrm{A}}$ receptors, and hence increases the total charge transfer in these synapses but not that of excitatory synapses [50]. To integrate physiological observations into neural models such as the Robinson model detailed in section 2.2, the anaesthetic action on synaptic receptors is modelled by $\alpha \rightarrow \alpha / p$ with $p \geq 1$ leading to a decrease of the decay rate constant $\alpha$, or equivalently, an increase in decay time constant of $\mathrm{GABA}_{\mathrm{A}}$ receptors [51]. The factor $p=1$ reflects absent anaesthetic action, i.e, the baseline condition. The model considers inhibitory synapses at excitatory neurons (factor $p_{1}$ ), and at inhibitory neurons (factor $p_{2}$ ) and at thalamic relay neurons (factor $\left.p_{3}\right)$.

To compute the power spectrum of the system, we consider the stationary state of Eq. (2), which obeys $d V_{a}(t) / d t=0$ where $V_{a}$ is taken from Eq. (2). Increasing the anaesthetic concentration, i.e. increasing the three factors $p_{1}, p_{2}$ and $p_{3}$ changes the stationary states dependent on the relation of these three factors, cf. Fig. 6. In (a) the two lower stationary states collide to a single state whereas in (b) the two upper states collide. This difference indicates two fundamentally different mechanisms which may yield different power spectra.

The power spectrum characterises small fluctuations about this stationary state. Assuming that excitatory activity generates the EEG, and by virtue of the specific choice of external input to relay neurons, the power spectrum of the EEG depends just on one matrix component of the Greens function [51] by

$$
P_{E}(\omega)=2 \kappa \sqrt{2 \pi}\left|\tilde{G}_{1,3}(\omega)\right|^{2}
$$

in which $\tilde{G}_{1,3}(\omega)$ is a matrix element of the $4 \times 4$ matrix 
a)

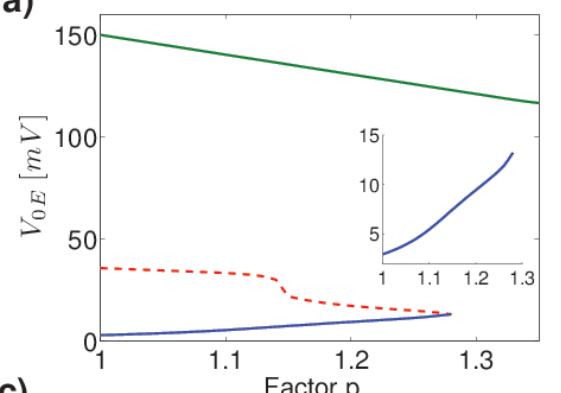

c)

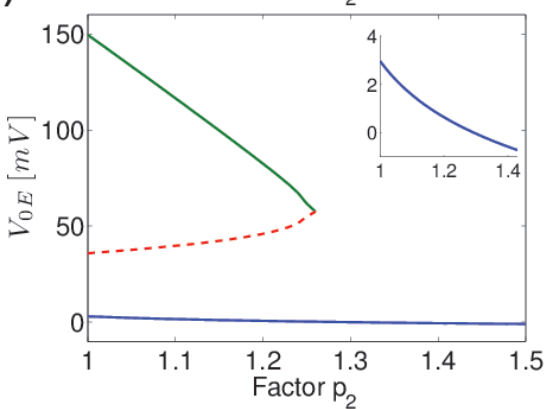

b)
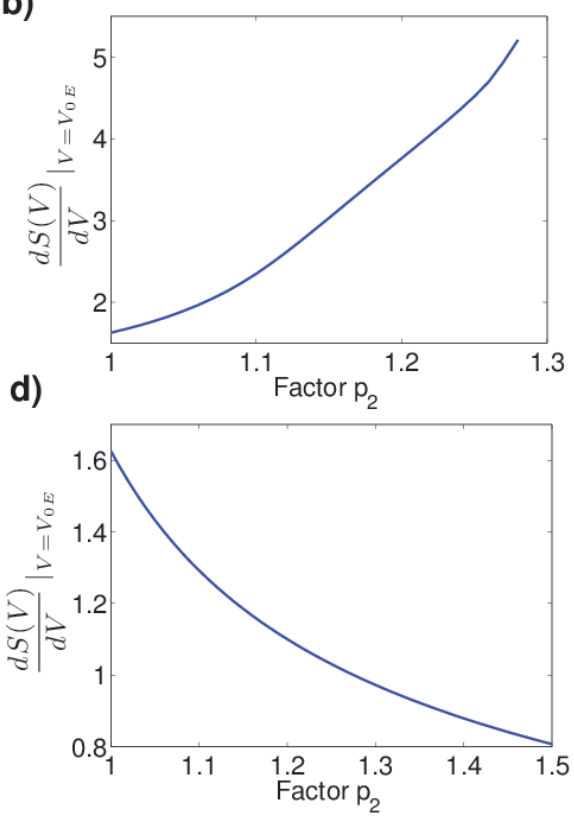

Fig. 6 The stationary states and the nonlinear gain $d S / d V$ computed at the lowest stationary state of pyramidal neurons $V_{E}$ subjected to the factor $p_{2}$. (a), (b) $p_{1}=p_{3}=1+0.3\left(p_{2}-1\right)$, (c), (d) $p_{1}=p_{2}=p_{3}$. We observe three states in (a) and (c) for $p_{2}=1$ where (a) the two lower states collide and (c) the two upper states collide. The center branch (dashed red) is linearly unstable, whereas the other branches are linearly stable. For clarification, the lower branches are shown in the insets. Parameters are $S_{\max }=250 \mathrm{~Hz}, V_{t h}=15 \mathrm{mV}, \gamma=0.08 \mathrm{mV}$ and $\sigma=10 \mathrm{mV}$.

$$
\tilde{G}(\omega)=\frac{1}{\sqrt{2 \pi}}\left[\begin{array}{cccc}
\tilde{L} \frac{\tilde{D}}{K_{11}}-K_{1} & -K_{2} & -K_{3} e^{-i \omega \tau} & 0 \\
-K_{4} & \tilde{L}-K 5 & -K_{6} & 0 \\
-K_{7} e^{-i \omega \tau} & 0 & \tilde{L} & -K_{8} \\
-K_{9} e^{-i \omega \tau} & 0 & -K_{10} & \tilde{L}
\end{array}\right]^{-1}
$$

with $\tilde{L}=(1+\mathrm{i} \omega / \alpha)(1+i \omega / \beta), \tilde{D}=(1+\mathrm{i} \omega / \gamma)^{2}$, and the constants $K_{i}, i=$ $1,2, \ldots, 11$ are proportional to the synaptic strengths and the nonlinear gains $\partial S / \partial V$ computed at the stationary state of the system.

Figure 7 shows the theoretical power spectrum $P_{E}$ in the baseline condition and after the administration of propofol for two different relations of the anaesthetic factors $p_{1}, p_{2}$ and $p_{3}$. At first we note that the spectra resemble well the spectrum obtained from experimental observations: increasing $p_{2}$ in a specific relation to the $p_{1}$ and $p_{3}$ yields increases in delta and theta power as well as more pronounced alpha oscillation with increased peak-frequency. The dynamical analysis of the model [30] reveals three resonances in the baseline condition, including an oscillatory resonance corresponding to the peak in the alpha-band and a pair of zero-frequency resonances. Increasing the anaesthetic concentration diminishes the 
damping rate of alpha resonances (and hence increases its magnitude) while its frequency increases. Hence increases in alpha power and its peak-frequency results from the approach of the system of an oscillatory instability [52]. Moreover, the two zero-frequency resonances collide and gradually increases in frequency leading to a magnitude increases in delta and theta power (solid red line in Fig. 7.)

a)

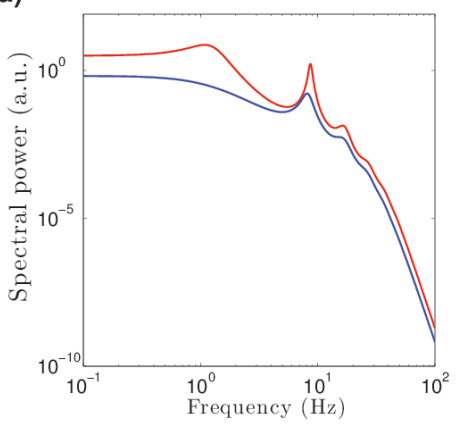

b)

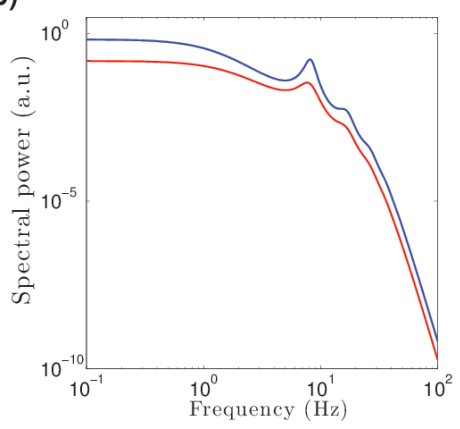

Fig. 7 Theoretical power spectrum in the baseline condition $\left(p_{1}=p_{2}=p_{3}=1.0\right.$ encoded in blue) and in the anesthesia condition (red line). (a) $p_{1}=p_{3}=1+0.3\left(p_{2}-1\right)$ and $p_{2}=1.15$ (red line) and (b) $p_{1}=p_{2}=p_{3}=1.15$ (red line).

Summarizing, previous studies $[30,51]$ have revealed the differential role of synaptic inhibition at GABAergic receptors located on the dendrites of different neural types. Moreover, synaptic potentiation within local cortical inhibitory neurons suffices to reproduce experimental observation in EEG during anesthesia [30, 52].

\section{Heterogeneous neural fields}

Previous experimental studies on the structure of biological neural networks and their connections, e.g., as the exciting work of Hellwig [53], reveals a large rather simple mesoscopic structure at the spatial scale of several hundreds of micrometers with an underlying more complex structure at smaller scales. For instance, [53] reveals a rather homogeneuous Gaussian or exponential connectivity distribution of single neurons in layers II and III in rat visual cortex on a scale of $\sim 500 \mu \mathrm{m}$ with an overlying complex connectivity structure at much shorter scales reflecting heterogeneous connections. Hence, a homogeneous model serves as a first approximation 
at larger spatial scales, whereas heterogeneous connections need to be considered in more realistic models.

Several previous theoretical studies have investigated the effect of heterogeneous connections in neural fields, primarily in the context of pattern formation [54-58]. Most of the previous studies consider the heterogeneous fields as small heterogeneous perturbations of homogeneous states. The subsequent paragraphs show a new approach taking into account the heterogeneous structure not as a limit of the homogeneous case.

Starting from the Amari equation (1), we expand the integral into a power series [19]

$$
\begin{aligned}
& \frac{\partial V(x, t)}{\partial t}+V(x, t) \\
& =\int_{\Omega} K_{1}(x, y) V(y, t) \mathrm{d} y+\int_{\Omega} \int_{\Omega} K_{2}(x, y, z) V(y, t) V(z, t) \mathrm{d} y \mathrm{~d} z,
\end{aligned}
$$

where the kernels $K_{1}$ and $K_{2}$ have to be reconstructed from experimental data.

\section{Event-related potentials}

In order to illustrate this, we combine a recently developed technique for nonlinear data analysis [18] with our new method for training heterogeneous neural fields [19] [19]. Figure 8 shows the grand average event-related potentials (ERP) $U_{n}(t), n=1 \ldots, N$ of $N=25$ recording electrodes from a language processing experiment of subject-object ambiguities in German [59]. The first column displays the control condition with a canonical subject-verb-object order: "Die Rednerin hat den Berater beim Kongress gesucht" ("The speaker has sought the advisor at the congress"), while the second column (Fig. 8(e-h)) shows ERPs for a non-canonical, yet in German grammatical, object-verb-subject order: "Die Rednerin hat der Berater beim Kongress gesucht" "The speaker has been sought by the advisor at the congress"). The panels (a) and (b) in the first row of Fig. 8 present the ERPs averaged over 14 subjects elicited by the disambiguating article "den" vs. "der" at time zero. Each trace shows the voltage of one of $N=25$ EEG electrodes. Comparing panels (a) and (b) in Fig. 8, one recognises a difference between conditions at about $600 \mathrm{~ms}$ after onset of the critical stimulus, known as the P600 ERP component.

\section{Heteroclinic orbits}

To analyze these spatiotemporal ERP patterns we apply our recently developed recurrence domain segmentation technique [18] whose results are shown in Fig. 8(c) and (d). This method computes the recurrence plots from the $N$-dimensional trajectories of each condition using a ball-size $\varepsilon>0$ as parameter. Here, we optimized $\varepsilon$ 


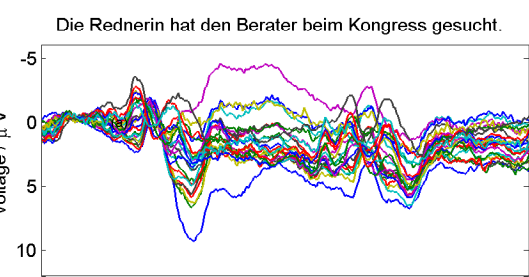

Die Rednerin hat der Berater beim Kongress gesucht.

(c)
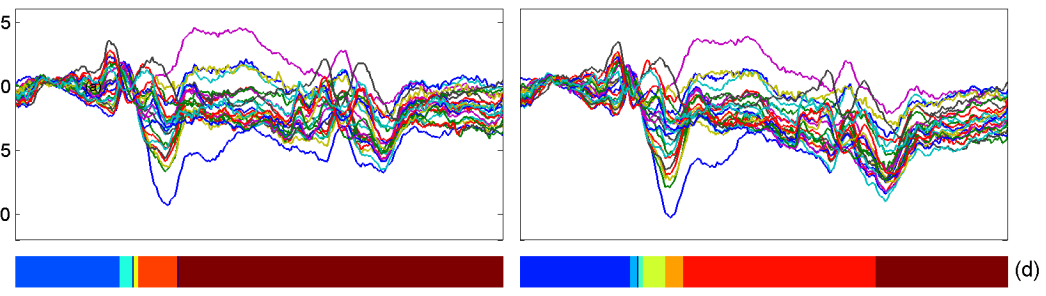

(e)

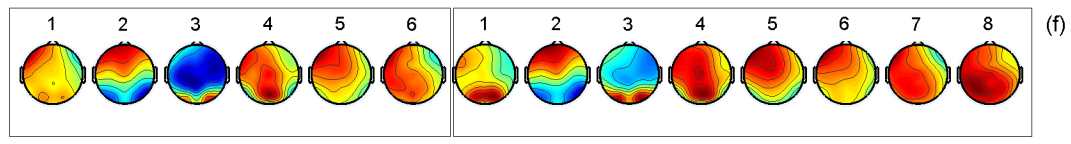

(g)

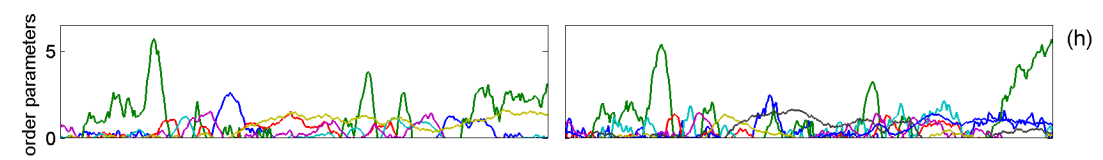

(i)

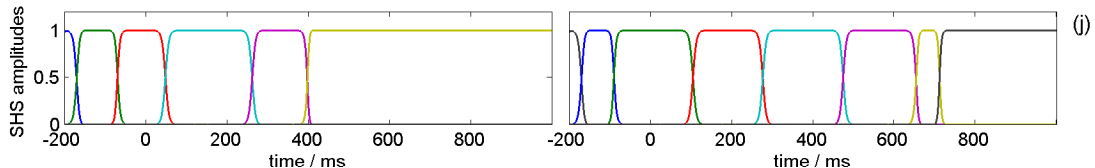

Fig. 8 Experimental event-related potentials (ERP) [59] in two experimental conditions (left and right column) and their interpretation as heteroclinic orbits. (a,b) ERPs of $N=25$ single EEG time series, (c,d) temporal sequence of extracted recurrence domains, (e,f) the spatial EEG activity distributions on the scalp (ERP components, seen from above, nose on top, back of head on bottom of the maps) averaged over the time windows of recurrence domains shown in panels (c,d), projections of the multivariate EEG signal (shown in panels $(\mathrm{a}, \mathrm{b})$ ) on the corresponding ERP component maps (shown in panels (e,f), (g,h) sequence of heteroclinic saddles (SHS) modeled by the Lotka-Volterra model.

according to a reasonable heuristics for obtaining a relatively low number of recurrence domains with relatively long dwell times and good (visual) discrimination of conditions which worked quite well for $\varepsilon=1.9 \mu \mathrm{V}$. Following [18], the recurrence plots are interpreted as rewriting grammars to replace large time indices by lower, recurrent, ones. These transformed time indices are plotted in Fig. 8(c) and (d) as different colors. The processing differences between conditions are clearly seen as different segments, indicating that ERP components, such as the P600, can be regarded as recurrence domains, or, in first approximation, as saddle nodes [60,61].

These saddles are estimated in Fig. 8(c) and (g) as follows: For each segment $k$ of Fig. 8(c) and (d) we compute its center of gravity, i.e. its temporal average, to obtain a spatial EEG activity distribution on the scalp, namely a spatial voltage distribution $U_{k}(z), k=1 \ldots, P$ with number of segments $P$ and where $z$ is the spatial location on the scalp. The patterns $U_{k}(z)$ are activity patterns extrapolated in space on the basis of the average ERP components which are discrete in space. They are shown in Fig. $8(\mathrm{e})$ and (f).

These distributions are assumed to originate from underlying neural patterns embed- 
ded in neural populations, e.g. sub-networks in neural populations. These underlying corresponding neural patterns may be called $V_{k}(x)$. Let us call $V(x, t)$ the neural population activity generating the EEG signal. Then the Moore-Penrose pseudoinverses $V_{k}(x)^{+}$of $V_{k}(x)$ form a bi-orthogonal basis with the patterns $V_{k}(x)$, such that the projections

$$
\xi_{k}(t)=\int_{\Omega} V_{k}(x)^{+} V(x, t) \mathrm{d} x
$$

serve as order parameters in a decomposition

$$
V(x, t)=\sum_{k} \xi_{k}(t) V_{k}(x) .
$$

The $\xi_{k}(t)$ are plotted in Figs. 8(g) and (h) where each trace depicts the projection onto one segment from the recurrence domain analysis in Figs. 8(c) and (d).

The dynamics of the order parameters $\left\{\xi_{k}\right\}$ is of high temporal complexity, indicating that a large number of time scales is involved in the ERP language processing dynamics. However, in a first approximation we assume that the recurrence domains are in fact isolated saddles that are connected along stable heteroclinic sequences (SHS). This can be modeled through winnerless competition in a network of Lotka-Volterra populations [62,63]. This assumption leads to the order parameter dynamics shown in $8(\mathrm{~g}, \mathrm{~h})$. From the growth rates $\sigma_{k}>0$, and interaction weights $\rho_{k j}>0, \rho_{k k}=1$, of the Lotka-Volterra dynamics and from the bi-orthogonal pattern systems $V_{j}(x), V_{k}(x)^{+}$, one can construct the kernels of the neural field equation (12) as

$$
\begin{aligned}
K_{1}(x, y) & =\sum_{k}\left(\sigma_{k}+1\right) V_{k}^{+}(y) V_{k}(x) \\
K_{2}(x, y, z) & =\sum_{k j} \rho_{k j} \sigma_{j} V_{k}^{+}(y) V_{j}^{+}(z) V_{k}(x),
\end{aligned}
$$

We observe that the kernel $K_{1}(x, y)$ describes Hebbian synapses between sites $y$ and $x$ trained with pattern sequence $V_{k}$ [19]. Interestingly, this memory storage mechanism resembles well the storage of patterns in a Bidirectional Associative Memory (BAM) [64].

Moreover, to our best knowledge the three-point kernel $K_{2}(x, y, z)$ has not been studied yet in the context of neural fields. It further generalizes Hebbian learning to interactions between three sites $x, y, z \in \Omega$. Interestingly, one can write

$$
\begin{aligned}
K_{1}(x, y) & =\sum_{k}\left(\sigma_{k}+1\right) K_{1, k}(x, y) \\
K_{2}(x, y, z) & =\sum_{j}\left[\sum_{k} \rho_{k j} K_{1, k}(x, y)\right] \sigma_{j} V_{j}^{+}(z) .
\end{aligned}
$$

with $K_{1, k}(x, y)=V_{k}^{+}(y) V_{k}(x)$. According to Eq. (13) the three-point kernel can be see as a linear superposition of two-point kernels weighted by the interaction weights $\rho_{k j}$ and the underlying stored patterns $V_{j}^{+}(z)$ and its corresponding growth rates $\sigma_{k}$. 
The additional dependence of the spatial location $z$ is reasonable in heterogeneous fields: the action at spatial location $x$ does not only depend on the activity in spatial location $y$ (as assumed in homogeneous systems), but may also depend on the path between $x$ and $y$ which in turn depends on the underlying memory structure. This argument motivates the presence of an additional spatial variable $z$, but does neither explain the terms in $K_{2}(x, y, z)$ nor its dependence on the specific choice of the heteroclinic dynamics (here a Lotka-Volterra system). Future work will attempt to elaborate more details on the presence of three-point kernels.

\section{Conclusion}

This chapter has presented some theoretical neural field studies describing the power spectra in homogeneous neural fields and the nonlinear dynamics in heterogeneous neural fields. In this context, we want to mention recent extensions of standard neural field models by incorporating single neuron properties [48, 65-67].

\section{Acknowledgements}

The authors thank Stefan Frisch and Heiner Drenhaus for conducting the ERP experiment. $\mathrm{AH}$ and $\mathrm{MH}$ acknowledge funding from the European Research Council for support under the European Union's Seventh Framework Programme (FP7/20072013) ERC grant agreement No.257253. PbG acknowledges financial support through a Heisenberg Fellowship of the German Research Foundation DFG (GR 3711/1-2).

\section{References}

1. P. Grassberger, Int. J. Theor. Phys. 25(9), 907 (1986)

2. L. Landau, E. Lifshitz, Fluid Mechanics (Butterworth-Heinemann, 1987)

3. C.G. Gross, Neuroscientist 8(5), 512 (2002)

4. N.E. Barraclough, D.I. Perrett, Phil. Trans. R. Soc. B 366(1571), 1739 (2011)

5. R.Q. Quiroga, L. Reddy, G. Kreiman, C. Koch, I. Fried, Nature 435(7045), 1102 (2005)

6. V.V. Vyazovskiy, K.D. Harris, Nature Rev. Neurosci. 14, 443 (2013)

7. B. Antkowiak, Anesthesiology 91, 500 (1999)

8. D. Belelli, N.L. Harrison, J. Maguire, R.L. Macdonald, M.C. Walker, D.W. Cope, J. Neurosc. 29(41), 12757 (2009)

9. P.C. Bressloff, J. Phys. A 45(3), 033001 (2012)

10. H. Wilson, J. Cowan, Kybernetik 13, 55 (1973)

11. S.I. Amari, Biol. Cybern. 27, 77 (1977)

12. D. Jancke, W. Erlhagen, H.R. Dinse, A.C. Akhavan, M. Giese, A. Steinhage, G. Schöner, J. Neurosci. 19(20), 9016 (1999)

13. S. Coombes, N.A. Venkov, L. Shiau, I. Bojak, D.T.J. Liley, C.R. Laing, Phys. Rev. E 76(5), 051901 (2007) 
14. A. Hutt, N. Rougier, Phys. Rev. E. 82, R055701 (2010)

15. A. Hutt, L. Zhang, J. Math. Neurosci. 3, 9 (2013)

16. A. Hutt, Phys. Rev. E 70, 052902 (2004)

17. A. Roxin, N. Brunel, D. Hansel, Phys.Rev.Lett. 94, 238103 (2005)

18. P. beim Graben, A. Hutt, Physical Review Letters 110(15), 154101 (2013). DOI 10.1103/PhysRevLett.110.154101

19. P. beim Graben, A. Hutt, EPJ Nonlin. Biomed. Phys. accepted (2014)

20. S. Coombes, M. Owen, Phys. Rev. Lett. 94, 148102 (2005)

21. O.D. Faugeras, J.D. Touboul, B. Cessac, Front. Comput. Neurosci. 3, 1 (2008)

22. N. Rougier, J. Vitay, Neural Networks 19(5), 573 (2006)

23. P. beim Graben, S. Rodrigues, Frontiers in Computational Neuroscience 6(100) (2013). DOI 10.3389/fncom.2012.00100

24. P. beim Graben, S. Rodrigues, in Neural Fields: Theory and Applications, ed. by S. Coombes, P. beim Graben, R. Potthast, J.J. Wright (Springer, Berlin, 2014)

25. S. Coombes, G. Lord, M. Owen, Physica D 178, 219 (2003)

26. S. Folias, P. Bressloff, SIAM J. Appl. Math 65, 2067 (2005)

27. V.K. Jirsa, H. Haken, Phys. Rev. Lett. 77(5), 960 (1996)

28. A. Hutt, Phys. Rev. E 75, 026214 (2007)

29. J. Wright, D. Liley, Behav. Brains Sc. 19, 285 (1996)

30. R. Hindriks, M.J.A.M. van Putten, Neuroimage 60, 2323 (2012)

31. J. Victor, J. Drover, M. Conte, N. Schiff, Proceed. Natl. Acad. Science USA 118, 15631 (2011)

32. P. Robinson, C.J.Rennie, D.L.Rowe, S.C.O'Connor, Human Brain Mapping 23, 53 (2004)

33. A. Hutt, M. Bestehorn, T. Wennekers, Network: Comput. Neural Syst. 14, 351 (2003)

34. A. Roxin, N. Brunel, D. Hansel, Prog. Theor. Phys. 161, 68 (2006)

35. F.M. Atay, A. Hutt, SIAM J. Appl. Dyn. Syst. 5(4), 670 (2006)

36. B. Doiron, M. Chacron, L.Maler, A. Longtin, J. Bastian, Nature 421, 539 (2003)

37. N.J. Berman, L. Maler, J. Exp. Biol. 202, 1243 (1999)

38. E. Ahissar, D. Kleinfeld, Cerebral Cortex 13, 53 (2003)

39. A. Hutt, C. Sutherland, A. Longtin, Phys.Rev.E 78, 021911 (2008)

40. P. Nunez, in Neocortical dynamics and human EEG rhythms, ed. by P. Nunez (Oxford University Press, New York-Oxford, 1995), pp. 475-533

41. M. Steyn-Ross, D. Steyn-Ross, J.W. Sleigh, D.T.J. Liley, Phys. Rev. E 60(6), 7299 (1999)

42. I. Bojak, D. Liley, Phys. Rev. E 71, 041902 (2005)

43. A. Hutt, J. Sleigh, A. Steyn-Ross, M.L. Steyn-Ross, Scholarpedia 8(8), 30485 (2013)

44. S. Ching, A. Cimenser, P.L. Purdon, E.N. Brown, N.J. Kopell, Proc. Natl. Acad. Sci. USA 107(52), 22665 (2010)

45. M.M. McCarthy, E.N. Brown, N. Kopell, J. Neurosci. 28(50), 13488 (2008)

46. A. Cimenser, P.L. Purdon, E.T. Pierce, J.L. Walsh, A.F. Salazar-Gomez, P.G. Harrell, C. Tavares-Stoeckel, K. Habeeb, E.N. Brown, Proc. Natl. Acad. Sci. USA 108(21), 8832 (2011)

47. M. Murphy, M.A. Bruno, B.A. Riedner, P. Boveroux, Q. Noirhomme, E.C. Landsness, J.F. Brichant, C. Phillips, M. Massimini, S. Laureys, G. Tononi, M. Boly, Sleep 34(3), 283 (2011)

48. A. Hutt, L. Buhry, submitted (2013)

49. B. Antkowiak, Brit. J. Anaesth. 89(1), 102 (2002)

50. A. Kitamura, W. Marszalec, J. Yeh, T. Narahashi, J. Pharmacol. 304(1), 162 (2002)

51. M. Hashemi, A. Hutt, submitted (2013)

52. A. Hutt, Front. Comp. Neurosci. 7, 2 (2013)

53. B. Hellwig, Biol. Cybernetics 82, 11 (2000)

54. V. Jirsa, J. Kelso, Phys.Rev.E 62(6), 8462 (2000)

55. Z.P. Kilpatrick, S.E. Folias, P.C. Bressloff, SIAM J. Applied Dynanmical Systems 7(1), 161 (2008)

56. S. Coombes, C. Laing, H. Schmidt, N. Svanstedt, J. Wyller, Discrete Contin. Dyn. Syst. A 32, $2951(2012)$

57. H. Schmidt, A. Hutt, L. Schimansky-Geier, Physica D 238(14), 1101 (2009)

58. P.C. Bressloff, Physica D 155, 83 (2001) 
59. P. beim Graben, S. Gerth, S. Vasishth, Cognitive Neurodynamics 2(3), 229 (2008)

60. A. Hutt, H. Riedel, Physica D 177(1-4), 203 (2003)

61. A. Hutt, International Journal of Bifurcation and Chaos 14(2), 653 (2004)

62. V.S. Afraimovich, V.P. Zhigulin, M.I. Rabinovich, Chaos 14(4), 1123 (2004)

63. M.I. Rabinovich, R. Huerta, P. Varona, V.S. Afraimovichs, PLoS Computational Biolog 4(5), e1000072 (2008)

64. B. Kosko, IEEE Transactions on Systems, Man and Cybernetics 18(1), 49 (1988)

65. A. Hutt, Cogn. Neurodyn. 6, 227 (2012)

66. J. Baladron, D. Fasoli, O. Faugeras, J. Touboul, J. Math. Neurosci. 2, 10 (2012)

67. P. Bressloff, SIAM J. Appl. Math 70, 1488 (2009) 